Originales

\title{
Radioterapia postoperatoria exclusiva en carcinoma de recto
}

J. Valencia Julve, R. Escó Barón, M. López Mata

\section{Resumen}

- Propósito: analizar los resultados del tratamiento radioterápico sin quimioterapia tras cirugía radical en cáncer rectal.

- Material y métodos: se analizaron los resultados de 20 pacientes tratados entre enero 94 y enero 97, por un cáncer rectal estadio B2-C. Se administró radioterapia postoperatoria exclusiva por no cumplir los requisitos para la administración de quimioterapia (edad >75 años, mala función hepática, renal o hematológica). Edad mediana: 71 años. Todos recibieron 50 Gy sobre volumen pélvico, con fraccionamiento estándar y técnica de cuatro campos, con fotones de $23 \mathrm{MV}(42,1 \%)$ o cobaltoterapia $(57,9 \%)$ y tras planificación bidimensional. Se analizaron las cifras de supervivencia, control de la enfermedad y toxicidad.

- Resultados: tras un seguimiento mediano de 76,64 meses, la supervivencia cáncer-específica y libre de enfermedad a 5 años fueron del $60,5 \%$ y del $58,1 \%$, respectivamente. La probabilidad de control local fue del $66,6 \%$ a 5 años y de control a distancia del 80,1\%. La incidencia de toxicidad aguda fue del 73,7\%, pero con ningún grado 3-4 de la RTOG, ni interrupciones de la radioterapia u hospitalización por toxicidad. La incidencia de toxicidad tardía grado 3 fue de un 5,3\%.

- Conclusiones: en aquellos pacientes con cáncer rectal en estadios B2-C en los que no se pueda administrar quimioterapia, debería ofrecerse la posibilidad de administrar radioterapia adyuvante exclusiva, dada su baja morbilidad y sus buenos resultados.

Palabras clave:

Radioterapia postoperatoria. Carcinoma rectal. Quimioterapia.

Oncología, 2004; 27 (5):297-306 


\section{Summary}

- Purpose: To analyze the results associated with postoperative radiotherapy without chemotherapy in rectal cancer.

- Material and methods: Twenty patients with B2-C stage rectal adenocarcinoma treated between January 1994 and January 1997 were analyzed. Surgery was followed by radiotherapy alone without chemotherapy because age $>75$ years, or renal, hepatic or hematotologic bad function. The median age was 71 years. All the patients received a dose of $50 \mathrm{~Gy}$ in the pelvic volume, with standard fractionation and a four-portal irradiation technique, by using $23 \mathrm{MV}$ photon beams $(42.1 \%)$ or 60 Co photons $(57.9 \%)$. Treatment planning was performed with two-dimensional dose-calculation algorithms.

- Results: With a median follow-up of 76.64 months, the actuarial cancer-specific and diseasefree survival rates were $60.5 \%$ and $58.1 \%$, respectively. Local control at 5 years was $66.6 \%$, and distant control $80.1 \%$. Acute toxicity incidence was $73.7 \%$. No grade 3-4 toxicity was produced, and it was not necessary to interrupt radiation therapy or hospitalization because of toxicity. Late grade 3 toxicity appeared in $5.3 \%$ of the patients.

- Conclusions: Patients with B2-C stage rectal cancer in which chemotherapy is not possible, can receive postoperative radiotherapy alone considering its low toxicity and good results.

Key words: Rectal carcinoma. Postoperative radiotherapy. Chemotherapy.

\section{Introducción}

Desde la conferencia de consenso del National Institute of Health', publicada en 1990, el tratamiento estándar del carcinoma rectal en estadios B2-3 y C según la clasificación modificada de Astler-Coller ${ }^{2}$, es la cirugía radical seguida de terapia combinada, con quimioterapia y radioterapia, en contraposición con la utilización aislada de cualquiera de las dos modalidades. Este consenso se alcanzó tras los buenos resultados del ensayo del GITSG ${ }^{3,4}$ y el del NCCTG $^{5}$, sobre pacientes con estadios patológicos T3-4 y/o con afectación ganglionar. No obstante, la generalización de la radioterapia ${ }^{6,7}$ y de la quimiorradioterapia preoperatoria $^{8-11}$, con excelentes resultados en términos de tasas de respuestas patológicas completas, cifras de subestadiaje y tasas de control local, nos hacen preveer un cambio rápido en la pauta de tratamiento del carcinoma rectal localmente avanzado, aunque el tratamiento adyuvante o postquirúrgico debe considerarse aún como estándar ${ }^{12-14}$.

Sin embargo, muchos de los pacientes que llegan a nuestros centros tras haber sido sometidos a una cirugía radical, no son subsidiarios de un tratamiento combinado con quimioterapia y radioterapia, bien por razones de edad o bien por no cumplir los criterios he- matológicos o de función hepática o renal para la administración de quimioterapia. En estos pacientes con cáncer de recto en estadios $\mathrm{B} 2-\mathrm{C}$, el riesgo de recidiva local es elevado, cifrándose hasta en un $68 \%$ en trabajos ya clásicos ${ }^{15,16}$, sin excisión mesorrectal total. En estos casos, aún sin poder administrar quimioterapia, la irradiación pélvica postquirúrgica puede ser un tratamiento indicado, cuando menos para aumentar el control local, y quizás con ello mejorar su supervivencia. Por ejemplo, Hoskins et a $\left.\right|^{17}$ encuentran un 9,5\% de fallo local en pacientes sometidos a radioterapia postquirúrgica frente a un $39 \%$ en un grupo tratado con cirugía exclusiva.

En este trabajo presentamos los resultados de un grupo de pacientes a los que no se les administró quimioterapia, pero sí radioterapia postoperatoria, por un carcinoma de recto en estadio B2-C en nuestro Servicio de Oncología Radioterápica del Hospital Clínico Universitario Lozano Blesa, de Zaragoza.

\section{Material y metodos}

\section{Pacientes y período de estudio}

El presente trabajo se basa en el análisis de 20 pa- 
cientes diagnosticados de un carcinoma primario rectal, tratados con radioterapia complementaria exclusiva en nuestro Servicio de Oncología Radioterápica, entre el 1 de enero de 1994 y el 1 de enero de 1997. Estos pacientes cumplían los siguientes criterios:

1. Diagnóstico de adenocarcinoma de recto, situado como máximo a $15 \mathrm{~cm}$. del margen anal, histológicamente confirmado.

2. Cirugía con intención curativa, mediante técnica de resección abdomino-perineal (R.A.P.), o resección anterior (R.A.), sin enfermedad residual macroscópica.

3. Estadios B2-C de la clasificación modificada de Astler-Coller ${ }^{2}$, es decir, con penetración tumoral a través de toda la pared rectal (pT3, pT4) y/o presencia de ganglios positivos en el estudio anatomopatológico ( $\mathrm{pN} 1, \mathrm{pN} 2)$, sin presentar metástasis a distancia (MO).

4. Buen estado funcional, con índice de Karnoffsky $\geq 70 \%$.

Durante este período, se había establecido un protocolo de tratamiento adyuvante combinando quimioterapia y radioterapia en todos los casos que cumplieran los criterios previos y además los siguientes:

- función renal adecuada (creatinina sérica $<1.1$ $\mathrm{mg} / \mathrm{dll}$ ).

- buena función hepática (bilirrubina total dentro de límites de normalidad, AST y ALT $<2$ veces el límite superior del centro).

- cifras de función hematológica dentro de los límites de la normalidad (leucocitos $>4.000 / \mathrm{mm}^{3}$ y plaquetas $>100.000 / \mathrm{mm}^{3}$.

- edad inferior a 75 años.

Los pacientes analizados en el presente estudio no cumplían alguno de estos cuatro últimos criterios o rehusaron recibir tratamiento quimioterápico adyuvante a cirugía.

Aquellos pacientes con tumores dobles o sincrónicos, enfermedades intercurrentes graves no controladas, mujeres embarazadas o en período de lactancia, o pacientes en los que se hubiese administrado radioterapia previa sobre volumen pélvico, fueron excluidos de la administración de tratamiento radioterápico.

\section{Tratamiento}

Tras el diagnóstico histopatológico de adenocarcinoma rectal y el diagnóstico clínico de enfermedad limitada a pelvis, todos los pacientes fueron sometidos a cirugía con intención radical, mediante resección en bloque de la lesión. Posteriormente, se analizaron el grado de penetración transmural y la presencia y número de ganglios metastatizados, para determinar el estadio postquirúrgico definitivo. Los pacientes diagnosticados de adenocarcinoma rectal en estadio B2-C de la clasificación modificada de Astler-Coller ${ }^{2}$ y que no cumplían los criterios de edad inferior a 75 años y/o cifras de función hepática, renal o hematológica compatibles con la administración de quimioterapia, fueron remitidos a nuestro Servicio de Oncología Radioterápica para valorar la indicación de irradiación pélvica.

La dosis total prescrita en el punto ICRU (intersección de los campos de tratamiento, coincidente con el isocentro del PTV) fue de 50 Gy en 25 fracciones, con fraccionamiento de 2 Gy/sesión, 5 sesiones/semana. Se decidió utilizar este fraccionamiento dado que no se administró quimioterapia como parte del tratamiento oncológico. La planificación se llevó a cabo mediante dosimetría computarizada bidimensional mediante radiografías ortogonales, sin utilización de imágenes TC en ningún caso y la distribución de isodosis fue calculada para cada paciente en el plano central transversal, después de que el oncólogo radioterápico determinara el PTV en dicho plano. No se realizó ninguna modificación para compensar las interrupciones del tratamiento radioterápico. Se utilizaron fotones procedentes de una unidad de Co-60 o Rx de $23 \mathrm{MV}$, y una técnica de cuatro campos en caja (box); todos los pacientes fueron colocados en decúbito prono y con la vejiga distendida. Se obtuvieron imágenes portales de los campos anterior y lateral el primer día de tratamiento.

El volumen de tratamiento incluyó lecho quirúrgico y cadenas ganglionares regionales a riesgo: los límites laterales en los campos antero-posterior y postero-anterior (AP/PA) fueron la escotadura pélvica, con un margen de 1-2 cms. El límite superior se situó $1,5 \mathrm{cms}$. por encima del promontorio sacro. Tras una resección anterior, el límite inferior de los campos se situó en el margen inferior de los agujeros obturadores, mientras que tras una resección abdomino-perineal el límite inferior se amplió hasta incluir toda la cicatriz perineal. Para los campos laterales el límite posterior se localizó 1,5-2 cms por detrás del margen anterior del sacro y el límite anterior o ventral en el margen posterior de la sínfisis púbica. Si las cadenas ilíacas externas fueron consideradas de riesgo, por afectación tumoral de órganos como próstata o vejiga, el límite ventral se situó en el margen anterior de la sínfisis púbica. En todos los casos se construyeron bloques personalizados para proteger tejidos sanos en los campos anteriores, posteriores y laterales. 


\section{Seguimiento y evaluación de toxicidad}

Durante el tratamiento radioterápico todos los pacientes fueron sometidos a revisiones semanales, con realización de analítica o pruebas complementarias sólo cuando se consideró necesario, pero no de forma rutinaria. En caso de aparición de toxicidad aguda grado 3 o superior según la escala de la RTOG ${ }^{18}$, la irradiación fue interrumpida temporalmente, hasta su resolución.

Tras la finalización de la irradiación, todos los pacientes fueron evaluados cada tres meses durante los dos primeros años y semestralmente hasta un mínimo de cinco años; a partir de ese momento, las revisiones fueron anuales. Al menos con una periodicidad semestral se realizaron radiografía de tórax, ecografía abdominal y analítica sanguínea y una vez al año TC abdomino-pélvico y fibroendoscopia. En caso de clínica sospechosa de metástasis a distancia se realizaban las exploraciones pertinentes, como TC cerebral o gammagrafía ósea. Tanto la toxicidad aguda como tardía fueron valoradas siguiendo los criterios de la EORTC/RTOG ${ }^{18}$.

\section{Diseño y análisis estadístico}

Se trata de un estudio prospectivo, no aleatorizado, llevado a cabo para evaluar la efectividad y tolerancia del tratamiento radioterápico como terapia adyuvante exclusiva en carcinoma rectal, en aquellos pacientes en los que no se administró quimioterapia. La recopilación de toda la información se realizó, mayoritariamente, a través de la revisión de las historias clínicas.

Todos los datos fueron introducidos en una base de datos (Access) y posteriormente analizados estadísticamente, procediéndose a un análisis descriptivo para los distintos factores pronósticos y características generales de los pacientes. Posteriormente se realizó un análisis de las tasas de toxicidad aguda y tardía aparecidas y de la supervivencia global, libre de enfermedad y cáncer específica siguiendo el método de Kaplan-Meier, así como de las tasas y funciones de riesgo de recidiva global, local y de metástasis a distancia. Para el análisis de recurrencia y supervivencia se consideró como fecha de inicio la del primer tratamiento, que en nuestro estudio fue la fecha de la cirugía y se han calculado los intervalos de confianza al 95\% (I.C. 95\%) de la mediana de supervivencia y tiempo a la recaída. Dado el escaso número de pacientes incluidos en la muestra, sólo fue posible analizar la influencia del estadio tumoral como factor pronóstico en los índices de supervivencia y control de la enfermedad. En este caso, para la comparación de las curvas de supervivencia se utilizó el test de log-rank y fue considerado un valor de $\mathrm{p} \leq 0,05$ para indicar significación estadística.

\section{Resultados}

Entre el 1 de enero de 1994 y el 1 de enero de 1997 fueron tratados en el Servicio de Oncología Radioterápica del Hospital Clínico Universitario "Lozano Blesa" de Zaragoza un total de 20 pacientes diagnosticados de un carcinoma rectal que no recibieron tratamiento quimioterápico por edad superior a 75 años (10 pacientes), cifras de función hepática, renal o hematológica no compatibles con la administración de quimioterapia (6 pacientes), o rehusar su administración (4 pacientes). Durante el seguimiento se perdió un paciente (5\%); por tanto, la muestra evaluada en este estudio ha quedado constituida por 19 pacientes, cuyas principales características quedan reflejadas en la Tabla I.

Desde el inicio del estudio en enero de 1994 hasta la fecha de la realización del análisis estadístico en enero de 2002, el seguimiento mediano para los pacientes supervivientes ha sido de 76,64 meses, con un seguimiento mínimo de 56 meses.

\section{Eventos}

En el momento de realizar el análisis estadístico, 12 pacientes habían fallecido, lo que representa un $63,2 \%$ del total, pero hemos de destacar que sólo 6 de las muertes $(31,6 \%)$ estuvieron relacionadas con el cáncer de recto, mientras los otros 6 pacientes fallecieron por otras causas, si bien uno de ellos había presentado previamente recidiva local de la enfermedad, siendo rescatada quirúrgicamente y no guardó relación con la causa de la muerte. Los seis fallecimientos no relacionados con el cáncer de recto fueron debidos a cuatro infartos agudos de miocardio, un accidente cerebro-vascular, y un tumor cerebral. Los 7 pacientes $(36,8 \%)$ vivos al finalizar el estudio estaban libres de tumor.

Hasta el cierre del estudio, 7 pacientes $(36,8 \%)$ fallaron en el control de la enfermedad en cualquier localización (Tabla II). Se ha considerado como recidiva local todo fallo aparecido tanto en la región presacra como en la anastomosis quirúrgica y en la region perineal, es decir, dentro de los campos de irradiación, mientras la recidiva en cadenas ganglionares no incluidas en los campos de radioterapia ha sido excluida de esta consideración y se ha considerado recidiva regional. La recurrencia local como cualquier componente de fallo apareció en 5 casos $(26,3 \%)$, cuatro a nivel presacro y una recidiva perineal. En 3 casos 
TABLA I

\begin{tabular}{|c|c|}
\hline \multicolumn{2}{|c|}{$\begin{array}{c}\text { Características de los pacientes y del tratamiento } \\
\text { administrado }\end{array}$} \\
\hline Característica & № pacientes (\%) \\
\hline $\begin{array}{ll}\text { Sexo } & \\
& \text { Hombre } \\
\text { Mujer }\end{array}$ & $\begin{aligned} & 11(57.9) \\
& 8(42,1)\end{aligned}$ \\
\hline Edad Mediana (Amplitud) & 71 (52-77) \\
\hline $\begin{array}{c}\text { Localización tumoral** } \\
\text { Tercio inferior } \\
\text { Tercio medio } \\
\text { Tercio superior } \\
\text { Estadio T }\end{array}$ & $\begin{array}{r}4(22,2) \\
9(50) \\
5(27,8)\end{array}$ \\
\hline $\begin{array}{l}\text { T2 } \\
\text { T3 } \\
\text { T4 }\end{array}$ & $\begin{array}{r}1(5.3) \\
14(73,3) \\
4(21,1)\end{array}$ \\
\hline $\begin{aligned} & \text { Estadio } N \\
& \text { N0 } \\
& \text { N1 } \\
& \text { N2 }\end{aligned}$ & $\begin{array}{r}11(57,9) \\
6(31,6) \\
2(10,5)\end{array}$ \\
\hline $\begin{array}{c}\text { Estadio (Astler-Coller) } \\
\text { B2 } \\
\text { B3 } \\
\text { C1 } \\
\text { C2 } \\
\text { C3 }\end{array}$ & $\begin{array}{r}10(52,6) \\
2(10,5) \\
1(5,3) \\
4(21,1) \\
2(10,5)\end{array}$ \\
\hline $\begin{array}{l}\text { Márgenes de resección* } \\
\text { Libres } \\
\text { Próximos o afectos } \\
\text { Invasión vascular/linfática* }\end{array}$ & $\begin{array}{r}17(94,4) \\
\quad 1(5,6)\end{array}$ \\
\hline $\begin{array}{c}\text { Invasión vascular/linfática* } \\
\text { Presente } \\
\text { Ausente }\end{array}$ & $\begin{array}{l}2(25) \\
6(75)\end{array}$ \\
\hline $\begin{array}{c}\text { Invasión perineural }{ }^{*} \\
\text { Presente } \\
\text { Ausente }\end{array}$ & $\begin{array}{r}0(0) \\
8(42,1)\end{array}$ \\
\hline $\begin{array}{c}\text { Grado diferenciación* } \\
\text { Grado } 1 \\
\text { Grado } 2 \\
\text { Grado } 3\end{array}$ & $\begin{array}{r}2(11,8) \\
13(76,5) \\
2(11,8)\end{array}$ \\
\hline $\begin{array}{l}\text { Tipo de resección } \\
\text { Anterior } \\
\text { Abdominoperineal } \\
\text { Unidad de tratamiento RT }\end{array}$ & $\begin{array}{r}10(52,6) \\
9(47,4)\end{array}$ \\
\hline $\begin{array}{c}\text { Unidad de tratamiento RI } \\
\text { Cobaltoterapia } \\
\text { Acelerador lineal }\end{array}$ & $\begin{array}{r}11(57,9) \\
8(42,1)\end{array}$ \\
\hline $\begin{array}{l}\text { Dosis de radioterapia (Gy) } \\
\text { Media (amplitud) } \\
\text { Duración de radioterapia }\end{array}$ & $49.47(48-50)$ \\
\hline $\begin{array}{l}\text { Mediana (rango) } \\
\leq 6 \text { semanas } \\
>6 \text { semanas } \\
\text { Interrupciones RT (no tóxicas) }\end{array}$ & $\begin{array}{r}6,14(5,2-10,2) \\
9(47,4) \\
10(52,6)\end{array}$ \\
\hline $\begin{array}{l}\text { No } \\
\text { Sí }\end{array}$ & $\begin{array}{r}1(5,3) \\
18(94,7)\end{array}$ \\
\hline
\end{tabular}

*para aquellos parámetros que no fueron analizados en todos los pacientes, se muestra el porcentaje en relación, no con la muestra total $(n=19)$, sino con el total de casos en los que se recogieron dichos parámetros.

RT: radioterapia.
TABLA ॥

\begin{tabular}{lcr}
\hline \multicolumn{3}{c}{ Distribución de la recidiva } \\
\hline Tipo de fallo & Frecuencia & $\%$ \\
\hline Ninguno & 12 & 63,1 \\
Local exclusivo & 3 & 15,8 \\
A distancia exclusivo & 1 & 5,3 \\
Local y a distancia & 2 & 10,5 \\
Regional, no local & 1 & 5,3 \\
\hline
\end{tabular}

$(15,8 \%)$ el fallo local fue exclusivo y en 2 casos $(10,5 \%)$ se combinó con metástasis a distancia. Se produjeron $3(15,8 \%)$ recidivas a distancia, todas ellas pulmonares: en un caso $(5,3 \%)$ como único fallo de la enfermedad y en 2 casos se combinó con fallo local. Además, apareció una metástasis ganglionar fuera de los campos de irradiación, sin fallo a otro nivel, por lo que fue considerada como fallo regional.

\section{Supervivencia y tasa de recurrencia}

La mediana de supervivencia global se situó en 42,05 meses (IC 95\%: 8,69-75,41). La supervivencia global actuarial a 3 y 5 años fue del $58 \%$ y el $41,4 \%$, respectivamente. Debido al elevado número de fallecimientos no relacionados con el cáncer de recto, se estimaron las cifras de supervivencia cáncer-específica a 3 y 5 años, las cuales fueron del $77,1 \%$ y del $60,5 \%$, respectivamente (Fig. 1), sin haberse alcanzado la mediana de supervivencia cáncer-específica. La supervivencia libre de recidiva a 3 años y a 5 años fue del $66,5 \%$ y del $58,1 \%$, respectivamente, sin alcanzarse tampoco en este caso la mediana de supervivencia.

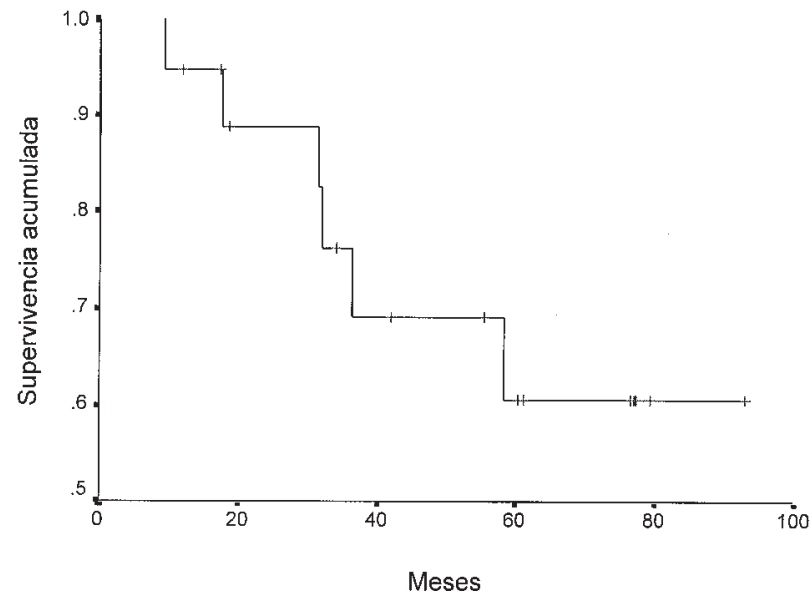

Fig 1. Supervivencia actuarial cáncer-específica para el total de la muestra $(n=19)$. 


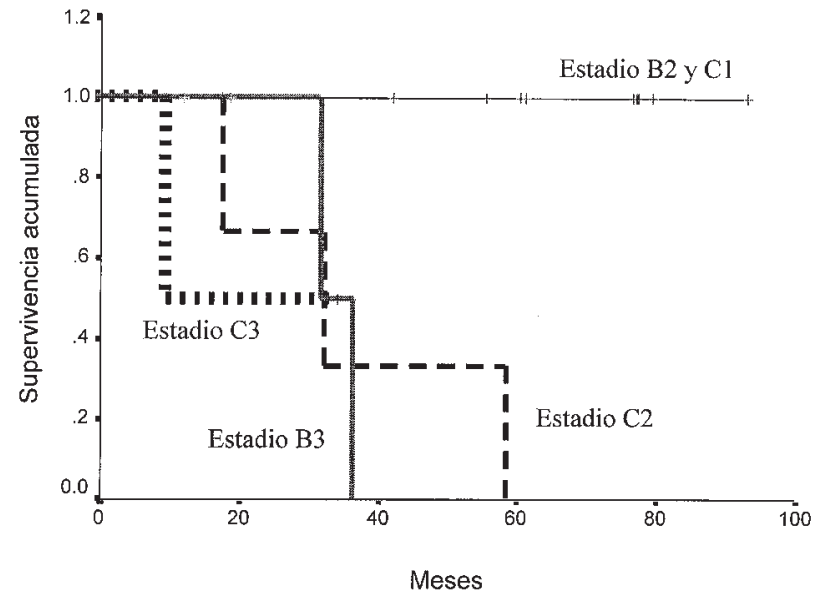

Fig 2. Supervivencia actuarial cáncer -específica en función del estadio tumoral según la clasificación modificada de Astar-Coller $(p=0,004)$.

En el análisis estadístico univariante, sólo se pudo analizar la influencia del estadio tumoral y de la presencia de afectación ganglionar en las cifras de supervivencia, debido a la escasez del número de pacientes y a que otros factores pronósticos, como la afectación de los márgenes de resección, grado de diferenciación tumoral, o presencia de afectación linfática o vascular solamente fueron recogidos en alguno de los registros. Destacar que todos los estadios B2 y $\mathrm{Cl}$ estaban vivos libres de enfermedad o habían fallecido por causas no relacionadas con su carcinoma rectal, por lo que el estadio tumoral se mostró como un factor predictivo para la supervivencia cáncer-específica $(p=0.004)$ y libre de recidiva $(p=0.034)$ (Fig. 2). Sin embargo, la presencia o ausencia de ganglios afectados por la enfermedad no mostró influencia en la mediana de supervivencia cáncer-específica (36 vs. 32 meses, $p=0.32$ ).

La probabilidad de recidiva global a 3 y 5 años fue del $33,5 \%$ y $41,8 \%$, respectivamente. La probabilidad de recidiva a distancia fue del $19,9 \%$ a 3 y 5 años, mientras la probabilidad de recidiva local fue del $23,7 \%$ a 3 años y del 33,3\% a 5 años (Fig. 3). En este caso, tampoco la presencia de afectación ganglionar mostró influencia estadísticamente significativa en la probabilidad de control local ( $80 \%$ a 5 años para NO vs. $36 \%$ para $\mathrm{N}+, p=0.22$ ), posiblemente por la escasez de pacientes a estudio. El estadio tumoral según la clasificación de Astler-Coller sí mostró influencia ( $p=0.006$ ) en la probabilidad de control local, ya que ninguno de los pacientes con estadios $\mathrm{B} 2$ y $\mathrm{Cl}$ presentaron fallo local, mientras que todos los B3 y la mitad de los C2 y C3 presentaron fallo a dicho nivel.

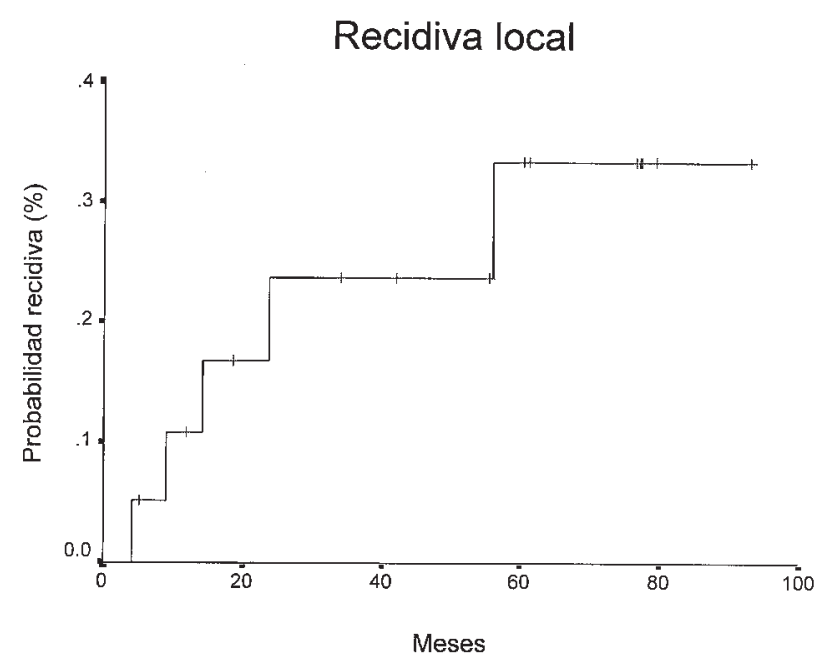

Fig 3. Probabilidad de recidiva local para el total de la muestra $(n=19)$.

\section{Toxicidad}

Se consideraron efectos secundarios agudos asociados al tratamiento radioterápico aquellos aparecidos durante los primeros 90 días tras el comienzo de la radioterapia, siguiendo las recomendaciones y la escala de toxicidad de la RTOG ${ }^{18}$. La toxicidad aguda aparecida, de cualquier grado, se muestra en la Tabla III. Como se puede extraer de dicha tabla, los efectos secundarios más frecuentes fueron la aparición de diarrea y de epidermitis, ambas con una incidencia global del $47,3 \%$, seguidas por la aparición de cistitis $(21 \%)$, presentándose de forma aislada o combinadas entre sí. La toxicidad aguda apareció tras la administración de una dosis media de radioterapia de $26 \mathrm{~Gy}$ (mediana 28 Gy, amplitud 10-43 Gy).

Respecto al grado de la toxicidad aguda aparecida, 5 pacientes $(26,3 \%)$ no presentaron ningún efecto secundario al tratamiento radioterápico, mientras los 14 restantes $(73,7 \%)$ presentaron toxicidad moderada,

TABLA III

\section{Efectos secundarios agudos de cualquier grado por} radioterapia

\begin{tabular}{lcc}
\hline Toxicidad aguda & Frecuencia & $\%$ \\
\hline Ninguna & 5 & 26,3 \\
Diarrea exclusiva & 3 & 15,8 \\
Cistitis exclusiva & 2 & 10,5 \\
Epidermitis exclusiva & 3 & 15,8 \\
Diarrea y epidermitis & 4 & 21,1 \\
Diarrea, epidermitis y cistitis & 2 & 10,5 \\
\hline
\end{tabular}


grado 2 de la RTOG, sin que ningún paciente presentara efectos tóxicos relevantes, grado 3-4 de la RTOG. La toxicidad aguda se resolvió en una media de 53 días (mediana de 14 días), sin que ningún paciente requiriera hospitalización ni la interrupción del tratamiento radioterápico para su resolución.

En cuanto a la toxicidad tardía, tan sólo aparecieron efectos secundarios de cualquier grado en 3 pacientes (15,8\%): 2 pacientes (10,5\%) presentaron cuadros de suboclusión intestinal a los 1,3 y 8,5 meses desde la finalización de la radioterapia y un paciente sufrió un cuadro de oclusión intestinal $(5,3 \%)$ a los 12,8 meses. No hubo ningún caso de clínica urinaria crónica. Por tanto, solamente un paciente $(5,3 \%)$ presentó toxicidad tardía severa, grado 3 de la RTOG ${ }^{18}$, que requirió tratamiento quirúrgico para solucionar el cuadro obstructivo.

\section{Discusión}

La cirugía sigue siendo el pilar fundamental en el tratamiento del carcinoma rectal, practicándose en aproximadamente un $80 \%$ de los pacientes afectos de estos tumores ${ }^{19}$. A pesar de los excelentes resultados obtenidos con la excisión mesorrectal total, con alrededor de un $7 \%$ de fallo local ${ }^{20}$, y su superioridad sobre la cirugía "convencional" 21, todavía no se ha generalizado su uso en nuestro medio, debido posiblemente a la necesidad de un entrenamiento específico del cirujano y a la mayor tasa de complicaciones postquirúrgicas descritas con esta técnica. Con la cirugía "convencional" la tasa de fallo local se sitúa entre un 5\% para los estadios $A$ y $B 1$ hasta un $95 \%$ para los estadios C3, como ya describieron Gunderson y Sosin ${ }^{16}$, lo que conlleva unas cifras de supervivencia a 5 años de aproximadamente un $50 \%$ para pacientes con estadios B y de un $30 \%$ para estadios $C^{22}$, con una tasa de recidiva local entre un 20 y un $40 \%$ en la mayoría de las series con cirugía sola.

Por ello, a finales de los años 80 comenzaron a publicarse diversos trabajos donde se utilizaba la radioterapia como tratamiento complementario a la cirugía en carcinoma de recto. Schild et a ${ }^{23}$ publicaron la experiencia de la clínica Mayo con 139 pacientes en los que se administró radioterapia postoperatoria tras resección completa por un carcinoma rectal con alto riesgo de recidiva pélvica. La dosis mediana de radioterapia sobre pelvis fue de 50 Gy y un $32 \%$ de los pacientes recibió además 5 -FU士semustina. De los 65 pacientes con R.A.P., en 43 de ellos se incluyó la cicatriz perineal en el campo de irradiación. Tras un seguimiento mediano de 4,2 años, la cifra de supervivencia global a 5 años fue del $59 \%$, la tasa de fallo local fue del $22 \%$, sin influencia de ningún factor (estadio B: $20 \%$, estadio C $28 \%$ ), y la tasa de fallo perineal fue del $18 \%$ en aquellos pacientes en los que no se incluyó la cicatriz perineal en los campos de radioterapia, mientras sólo fue de un $2 \%$ en los pacientes en los que sí se incluyó ( $p=0.01)$. La tasa de fallo a distancia fue del $42 \%$. Hubo un $6 \%$ de oclusiones intestinales, requiriendo cirugía en un 3,5\% del total de los pacientes; no hubo muertes atribuibles al tratamiento adyuvante.

Otro trabajo ${ }^{17}$ retrospectivo, anterior al expuesto anteriormente, comparaba un grupo de 95 pacientes con cáncer rectal en estadios B2-C que recibieron radioterapia adyuvante (dosis media de $50.4 \mathrm{~Gy}$ ), con otros 105 pacientes con los mismos estadios tumorales tratados exclusivamente con cirugía potencialmente curativa; en ningún paciente se administró quimioterapia. En el grupo de pacientes con cirugía sola, la incidencia de fallo local fue del $39 \%$, mientras en el grupo irradiado el fallo local se produjo en 9 de los 95 pacientes $(9,5 \%)$, mientras el fallo a distancia ocurrió en el $30,5 \%$ de los pacientes. En el subgrupo de pacientes con ganglios negativos el porcentaje de fallo local fue del $6 \%$. A tres años se observó una reducción significativa en la tasa de recurrencia local en todos los estadios, excepto los C3, pero en cuanto a la supervivencia solamente hubo una ventaja estadísticamente significativa en los pacientes con tumores en estadio B3 ( $83 \%$ vs. $57 \%$ ).

Bentzen et al ${ }^{24}$ intentaron establecer subgrupos de pacientes con factores de alto riesgo de recidiva tras cirugía, que se beneficiarían selectivamente, en cuanto a control local y supervivencia, al añadir radioterapia. Estos autores recogen los datos de 468 pacientes con estadios $\mathrm{B}$ y $\mathrm{C}$, randomizados a recibir cirugía exclusiva o seguida de radioterapia: tras un seguimiento mínimo de 5 años no hubo beneficio significativo con el uso de radioterapia postoperatoria considerando todos los pacientes, pero en pacientes de alto riesgo (estadios C con invasión perineural y/o vascular, afectación de márgenes, a menos de $10 \mathrm{cms}$ de margen anal, con afectación de órganos vecinos) hubo una mejoría significativa en el control local a 5 años (40\% vs. $11 \%, p=0.03$ ), con impacto en la supervivencia global, aunque sin llegar a la significación estadística $(23 \%$ vs. $0 \%)$, posiblemente por el escaso número de pacientes en este subgrupo y el alto índice de recurrencia a distancia.

Por tanto, la radioterapia postoperatoria sola no ha demostrado un aumento significativo de la supervivencia en ninguno de los trabajos publicados $25-27$, aún disminuyendo la tasa de recidivas locales ${ }^{28,29}$. Por ello, y dado el gran porcentaje de pacientes que mueren con enfermedad a distancia, se empezó a investigar el tra- 
tamiento combinado quimiorradioterápico como adyuvante a la cirugía frente a la radioterapia postquirúrgica aislada. Este tratamiento se convirtió en el estándar en el cáncer de recto tras los dos ensayos randomizados $^{3-5}$, llevados a cabo por el Gastrointestinal Tumor Study Group (GITSG) ${ }^{3,4}$ y el North Central Cancer Treatment Group (NCCTG) y la Clínica Mayo ${ }^{5}$. Los mejores resultados en términos de control global, tiempo hasta la recidiva $y$, en el segundo de ellos también con una mejoría significativa en las cifras de supervivencia global, llevaron a la conferencia de consenso del $\mathrm{Na}$ tional Institute of Health' en 1990, donde la recomendación final es que el tratamiento estándar en el carcinoma rectal es la combinación postoperatoria de quimiorradioterapia. Este criterio, como exponen en sus respectivos trabajos Heriot ${ }^{30}$, Bosset $^{13}$ y Glimelius ${ }^{12}$, continúa en vigor, al menos hasta que los ensayos randomizados aún abiertos ${ }^{31-33}$, que comparan su administración frente a la prequirúrgica, nos den la respuesta definitiva, a pesar de sus excelentes resultados cuando se compara con la cirugía aislada $8,11,34,35$.

Sin embargo, ocasionalmente nos encontramos con pacientes que no han podido recibir quimioterapia tras la cirugía, debido, bien a que los criterios bioquímicos mínimos que garantizan una función hepática, renal y hematológica suficientes para su administración no se cumplen, o bien por razones de edad, la cual puede ocasionar una peor tolerancia a la quimioterapia. En este subgrupo de pacientes que reciben como tratamiento inicial una cirugía potencialmente radical sobre su carcinoma rectal y que no cumplen los criterios necesarios para la administración "segura" de quimioterapia, muchas veces nos preguntamos: ¿̇y merece la pena administrar radioterapia? ¿cómo tolerará la radioterapia con esa edad, o con esas cifras en la analítica?

Para darnos respuestas a estas preguntas, decidimos analizar la efectividad y la tolerancia al tratamiento radioterápico en un grupo de pacientes tratados postoperatoriamente por un carcinoma rectal. En este grupo, la supervivencia global a 5 años fue del $41,4 \%$, una cifra excesivamente baja, motivada por el elevado número de fallecimientos no relacionados con el cáncer de recto, concretamente la mitad de todos los producidos hasta el cierre del estudio. Por ello se estimó la supervivencia cáncer-específica, que se situó en el $60,5 \%$ a 5 años, cifra mucho más acorde con los datos de la literatura, como el $59 \%$ de Schild et a ${ }^{23}$ aún asociando quimioterapia en un $32 \%$ de los pacientes, y el $60 \%$ de Hoskins et a ${ }^{17}$ en el grupo asociando cirugía y radioterapia postoperatoria. También esta cifra de supervivencia es similar a las obtenidas en diversos trabajos combinando radioterapia y quimioterapia postoperatorias, como en un estudio propio $^{36}$, recientemente publicado, sobre 122 pacientes en estadios B2-C y en los que la supervivencia cáncer-específica se situó en un $60,5 \%$ a 5 años, o el trabajo de Ma y Phang ${ }^{37}$, también con un $60 \%$ a 5 años. Por tanto, vemos como, desde el punto de vista de la supervivencia a largo plazo relacionada con el cáncer rectal, parece que sí merece la pena administrar radioterapia postoperatoria en este subgrupo de pacientes en los que no se administra quimioterapia.

Cuando nos centramos en la probabilidad de control de la enfermedad, vemos cómo la probabilidad de recidiva a distancia (19,9\% a 3 y 5 años), es incluso inferior a la recogida en la literatura, que se situó en un $42 \%$ en el trabajo de Schild et a $\left.\right|^{23}$ y en un $30,5 \%$ en el de Hoskins et al ${ }^{17}$, ambos trabajos con la mayoría de pacientes recibiendo sólo radioterapia tras la cirugía. Pero también se sitúa por debajo del $26 \%$ a 5 años del ensayo del GITSG ${ }^{3}$, del $29 \%$ del trabajo de Lupatelli et al ${ }^{38}$ y del $32.6 \%$ de nuestro estudio previo $^{36}$, todos estos trabajos con radioquimioterapia postoperatoria. Probablemente esta excelente tasa de control a distancia se deba al alto porcentaje de pacientes en estadio B2, un $56,2 \%$ de la muestra, con menor riesgo de metástasis fuera de la pelvis.

Mientras, la probabilidad de recidiva local, del $33,3 \%$ a 5 años, aunque similar, también es ligeramente inferior al $34,6 \%$ hallado por nuestro grupo en el estudio previo combinando radioquimioterapia ${ }^{36}$, aunque notablemente superior al $22 \%$ obtenido por Schild et a ${ }^{23}$ y al 9,5\% recogido en el trabajo de Hoskins et a ${ }^{17}$, sólo con radioterapia postquirúrgica. No obstante, hemos de señalar que estos dos últimos resultados son excepcionalmente buenos, cuando los comparamos con trabajos en los que se administró radioquimioterapia tras la cirugía, como el ensayo del $\operatorname{NCCTG}^{5}\left(13,5 \%\right.$ a 5 años), el del GITSG $^{3}$ (11\% a 5 años), o el trabajo de Lupatelli et al ${ }^{38}$ ( $14 \%$ a 5 años).

En nuestro trabajo, la presencia de afectación ganglionar no mostró influencia significativa en la probabilidad de control local, a pesar de existir una gran diferencia porcentual, $80 \%$ a 5 años para NO vs. $36 \%$ para $\mathrm{N}_{+}$, debido, probablemente a lo corto de la muestra, aunque este hallazgo coincide con el de los trabajos de Schild et a ${ }^{23}$ y el de Hoskins et al ${ }^{17}$, ya que en ninguno de ellos aparece diferencia en el control local en función de la afectación ganglionar. Sin embargo, el estadio tumoral según la clasificación de Astler-Coller sí mostró influencia ( $p=0.006$ ) en la probabilidad de control local en nuestro estudio, ya que ninguno de los pacientes con estadios $\mathrm{B} 2$ y $\mathrm{C} 1$ presentaron fallo local, mientras que todos los B3 y la mitad de los C2 y C3 presentaron fallo a dicho nivel. Estos datos también son coincidentes con los de nuestro trabajo 
previo con radioquimioterapia ${ }^{36}$, donde los estadios B3, C2 y C3 presentaban cifras de recurrencia local alrededor de un $50 \%$ frente al $13,4 \%$ de los estadios B2 y al $0 \%$ de los $\mathrm{C}$. También Hoskins et al ${ }^{17}$, sólo con radioterapia postoperatoria, encontraron esta influencia del estadio tumoral en la tasa de control local, excepto para los estadios C3.

En cuanto a la tolerancia al tratamiento radioterápico, podemos decir que ésta fue excelente, ya que ningún paciente presentó toxicidad relevante, grados 3-4 de la RTOG ${ }^{18}$, ni fue necesario interrumpir el tratamiento radioterápico o ingresar al paciente para la resolución de los efectos tóxicos. Los efectos secundarios más frecuentes fueron la diarrea y la epidermitis, con un $47 \%$ de incidencia, cifra inferior al $55 \%$ recogida en nuestro trabajo con radioquimioterapia ${ }^{36}$. Además, en este trabajo ${ }^{36}$ se recogió un $20,5 \%$ de pacientes con toxicidad grado 3 de la RTOG ${ }^{18}$, en los que además se debió interrumpir el tratamiento radioterápico, con 4 pacientes requiriendo hospitalización. Estas cifras muestran la peor tolerancia del tratamiento radioquimioterápico combinado frente a la radioterapia sola, a pesar de que teóricamente este grupo de pacientes eran más lábiles por razones de edad o condición física. Las cifras de toxicidad tardía fueron similares a las obtenidas con la combinación de radioquimioterapia: 2 pacientes $(10,5 \%)$ presentaron cuadros de suboclusión intestinal, frente al $9 \%$ de nuestro trabajo con radioquimioterapia ${ }^{36}$, pero sólo un paciente $(5,3 \%)$ sufrió un cuadro de oclusión intestinal requiriendo cirugía, frente al 8,2\% del estudio con radioquimioterapia. Estos datos son también similares a los recogidos por Schild et a ${ }^{23}$, con un $6 \%$ de oclusiones intestinales, requiriendo cirugía en un 3,5\% del total de los pacientes.

Aunque sin poder llegar a una conclusión tras el estudio de este pequeño grupo de pacientes, a los que no se les administró quimioterapia tras cirugía curativa sobre su tumor rectal, nosotros consideramos que, en estos casos, sí se debe ofrecer la posibilidad de administrar radioterapia pélvica, dada su baja morbilidad, tanto aguda como tardía, y los buenos resultados en términos de control de la enfermedad y de supervivencia relacionada con dicho tumor rectal.

Correspondencia:

Dr. J. Valencia Julve

Servicio de Oncología Radioterápica

Hospital Clínico Universitario

C/San Juan Bosco, 15

E-50009 Zaragoza

E-mail: jvalenciaj@hotmail.com

\section{Bibliografía}

1. National Institute of Health Consensus Conference. Adjuvant therapy for patients with colon and rectal cancer. JAMA 1990; 264:1444-9.

2. Astler VB, Coller FA. The prognostic significance of direct extension of carcinoma of the colon and rectum. Ann Surg 1954; 139:846-50.

3. Gastrointestinal Tumor Study Group: Prolongation of the disease-free interaval in surgically treated rectal carcinoma. N Engl J Med 1985; 312:1465-72.

4. Douglass HO Jr, Moertel CG, Mayer RJ, et al. Survival after postoperative combination treatment of rectal cancer [cartal. N Engl J Med 1986; 315:1294.

5. Krook JE, Moertel CG, Gunderson LL, et al. Effective surgical adjuvant therapy for high-risk rectal carcinoma. $\mathrm{N}$ Engl J Med 1991; 324:709-15.

6. Swedish rectal cancer trial. Improved survival with preoperative radiotherapy in resectable rectal cancer. $\mathrm{N}$ Engl J Med 1997; 336:980-7.

7. Kapiteijn E, Marijnen AM, Nagtegaal ID, et al. Preoperative radiotherapy combined with total mesorrectal excision for resectable rectal cancer. N Engl J Med 2001; 345:638-46.

8. Janjan NA, Khoo VS, Abbruzzese J, et al. Tumor downstaging and sphincter preservation with preoperative chemoradiation in locally advanced rectal cancer: the MD Anderson Cancer Center experience. Int J Radiat Oncol Biol Phys 1999; 44:1027-38.

9. Bosset JF, Magnin V, Maingon P, et al. Preoperative radiochemotherapy in rectal cancer: long-term results of a phase II trial. Int J Radiat Oncol Biol Phys 2000; 46(2):323-7.

10. Calvo FA, Gómez-Espí M, Díaz-González JA, et al. Pathologic downstaging of T3-4 Nx rectal cancer after chemoradiation: 5-fluorouracil vs. Tegafur. Int J Radiat Oncol Biol Phys 2001; 51(5):1264-70.

11. Grann A, Feng C, Wong D, et al. Preoperative combined modality therapy for clinically resectable UT3 rectal adenocarcinoma. Int J Radiat Oncol Biol Phys 2001; 49(4):987-95.

12. Glimelius B. Chemoradiotherapy for rectal cancer. Is there an optimal combination?. Ann Oncol 2001; 12(8):1039-45.

13. Bosset JF, Mantion G, Lorchel F et al. Adjuvant and neoadjuvant radiation therapy for rectal cancer. Semin Oncol 2000, 27(5 Suppl):60-5.

14. Minsky BD. Adjuvant radiation therapy for rectal cancer: is there finally an answer? Lancet $2001 ; 358: 1285-6$.

15. Rich T, Gunderson LL, Lew R. et al. Patterns of recurrence of rectal cancer after potentially curative surgery. Cancer 1983; 52:1317-29.

16. Gunderson LL, Sosin H. Areas of failure found at reoperation (second or symptomatic look) following curative surgery for adenocarcinoma of the rectum: Clinicopat- 
hologic correlation and implications for adjuvant therapy. Cancer 1974; 34:1278-92.

17. Hoskins RB, Gunderson LL, Dorosetz DE, et al. Adjuvant postoperative radiotherapy in carcinoma of the rectum and rectosigmoid. Cancer 1985; 55:61-70.

18. Rubin P. Special issue: Late effects of normal tissues consensus conference, including RTOG/EORTC SOMA scales. Int J Radiat Oncol Biol Phys 1995; 31:1035-360.

19. Nelson H, Petrelli N, Carlin A, et al. Guidelines 2000 for Colon and Rectal Cancer Surgery. J Natl Cancer Inst 2001; 93:583-96.

20. Soreide $O$, Norstein J. Local recurrence after operative treatment of rectal carcinoma: a strategy for change. J Am Coll Surg 1997; 184:84-92

21. Arbman G, Nilsson E, Hallbook O, Sjodahl R. Local recurrence following total mesorectal excision for rectal cancer. Br J Surg 1996; 83:375-9.

22. Boyle P. Some recent developments in the epidemiology of colorectal cancer. En Bleiberg $\mathrm{H}$, Rougier P, Wilke HJ (eds): Management of colorectal cancer. London, UK, Martin Dunitz 1998, pp 19-34.

23. Schild SE, Marterson JA Jr, Gunderson LL, et al. Postoperative adjuvant therapy of rectal cancer: An analysis of disease control, survival and prognostic factors. Int J Radiat Oncol Biol Phys 1989; 17:55-62.

24. Bentzen SM, Balslev I, Pedersen M, et al. Time to locoregional recurrence after resection of Dukes $B$ and $C$ colorectal cancer with or without adjuvant postoperative radiotherapy: A Multivariate regression analysis. $\mathrm{Br} \mathrm{J}$ Cancer 1992; 65:102-7.

25. Mohiuddin M, Derler J, Marks G, et al. Results of adjuvant radiation therapy in cancer of the rectum. Cancer 1985; 55:350.

26. Tepper JE, Cohen AM, Wood WC, et al. Postoperative radiation therapy of rectal cancer. Int J Radiat Oncol Biol Phys 1987; 13:5.

27. Vigliotti A, Rich TA, Romsdahl MM, et al. Postoperative adjuvant radiotherapy for adenocarcinoma of the rectum and rectosigmoid. Int J Radiat Oncol Biol Phys 1987; 13:999.

28. Medical Research Council Rectal Cancer Working Party: Randomised trial of surgery alone versus surgery follo- wed by radiotherapy for mobile cancer of the rectum. Lancet 1996; 348(9042): 1610-4.

29. Treurniet-Donker AD, van Putten WL, Wereldsma JC, et al. Postoperative radiation therapy for rectal cancer: An interim analysis of a prospective, randomized multicenter trial in The Netherlands. Cancer 1991; 67:2042.

30. Heriot AG, Kumar D. Adjuvant therapy for resectable rectal and colonic cancer. Br J Surg 1998; 85(3):300-9.

31. Hyams DM, Mamounas EP, Petrelli $N$, et al. A clinical trial to evaluate the worth of preoperative multimodality therapy in patients with operable carcinoma of the rectum. A progress report of national surgical adjuvant breast and bowel project protocol R-03. Dis Colon Rectum 1997; 40:131-9.

32. Schick CH, Rodel C, Raab R, et al. Introduction of a prospective randomised trial: adjuvant and neoadjuvant radiochemotherapy in rectal cancer. Eur J Surg Oncol 1998; 24:286(abstr).

33. Saver R and German for the German Rectal Cancer Group. Adjuvant versus neoadjuvant combined modality treatment for locally advanced rectal cancer: first results of the German rectal cancer study (CAO/ARO/AIO94). Int J Radiat Oncol Biol Phys 2003; 57(2):S124S125.

34. Cammà C, Giunta M, Fiorica F, Pagliaro L, Craxi A, Cottone $M$. Preoperative radiotherapy for resectable rectal cancer. A meta-analysis. JAMA 2000;284(8):1008-15.

35. Colorectal Cancer Collaborative Group. Adjuvant radiotherapy for rectal cancer: a systematic overwiew of 8507 patients from 22 randomised trials. Lancet 2001; 358:1291-304.

36. Valencia J, Escó R, Sanz J, Burillo M, Mateo P, Lainez C. Tratamiento adyuvante del carcinoma de recto. Rev Oncol 2003; 5(9):524-36.

37. Ma R, Phang PT. Results of curative surgery and postoperative chemoradiation for rectal adenocarcinoma in British Columbia, 1985 to 1994. Can J Surg 2001; 44(5):377-82.

38. Lupattelli M, Maranzano E, Bellavita R, et al. Adjuvant radiochemotherapy in high-risk rectal cancer results of prospective non-randomized study. Tumori 2001; 87(4):239-47. 